This is an original manuscript of an article published by ELSEVIER in Journal of Composites Part B: Engineering 178 (2019) on 19 Sep. 2019, available online at: https://www.sciencedirect.com/science/article/pii/S1359836819332913?via\%3Dihub DOI: https://doi.org/10.1016/j.compositesb.2019.107461 


\title{
Evaluation of debonding in CFRP-epoxy adhesive single-lap joints using eddy current pulse-compression thermography
}

\author{
Q. Yi ${ }^{1}$, G.Y. Tian ${ }^{1}$, B. Yilmaz ${ }^{2}$, H. Malekmohammadi ${ }^{3}$, S. Laureti ${ }^{3}$, M. Ricci ${ }^{4}$, E. Jasiuniene ${ }^{2}$ \\ ${ }^{I}$ Department of Electrical and Electronic Engineering, Newcastle University, Merz Court, NE1 7RU, Newcastle upon Tyne, UK \\ ${ }^{2}$ Ultrasound Research Institute, Kaunas University of Technology, Kaunas, Lithuania \\ ${ }^{3}$ Department of Engineering, University of Perugia, Polo Scientifico didattico di Terni, Strada di Pentima 4, 05100 Terni, Italy \\ ${ }^{4}$ Department of Informatics, Modeling, Electronics and System Engineering, University of Calabria, Rende, 87036, Italy
}

\begin{abstract}
Contaminations arising during the manufacturing or usage of Carbon Fiber Reinforced Plastic (CFRP)-epoxy adhesive single-lap joints can drastically affect the mechanical performance of the structure and eventually lead to its catastrophic failure. Eddy current stimulated thermography has been recently proposed for detecting and characterizing defects in composite materials. The characterization of debonding in adhesive single-lap joints in composites by eddy current stimulated thermography can be challenging due to the anisotropic thermal diffusion caused by the anisotropic conductivity and multi-layer nature of the composites. This work tackles the challenge of detecting the interface contamination by combining eddy current pulsecompression thermography and image post-processing algorithms. The impulse responses from samples containing either brass, release film or release agent contaminations obtained through pulse-compression combined with Eddy Current Pulsed Thermography (ECPT). Non-uniform heating patterns are removed by partial least square technique. Then, the time instants containing meaningful information about the contaminated interface layer are inferred by comparing each norm of kernel principal component over suitable time windows. The evaluation of contamination depths and properties are conducted in selected time windows by principal component analysis and time-phase analysis. Results proved that release agent contamination plays the minor role in changing the electrical and thermal properties of the single-lap joints compared with brass and release film.
\end{abstract}

Keywords: Eddy Current Pulsed Thermography (ECPT), Pulse-compression, Adhesive joints, CFRP, Kernel Principal Component Analysis, Debonding

\section{Introduction}

CFRPs are extensively used in the aerospace industry owed to their extraordinary properties such as high strength to weight ratio, great thermal stability, and high resistance over impacts [1]. Adhesive bonding is one of the most advantageous joining technologies in composites material because the mechanical properties of such joints are significantly better than those achievable with other joint designs e.g. rivet. This can be attributed to their reduction of peel stress and interfacial shear stress [2]. Moreover, the adhesive joints are relatively simple to realize and cost-effective. The major advantages of the adhesive bonded joints include the reduction of local delamination, prevention of galvanic corrosion due to the fasteners, significant reduction in weights of the joints and a smooth external surface of the final component [3]. However, the risk of trapped air, dust, release film, release agent or other contaminations occurring during the manufacturing process can lead to the premature failure of the component, when they are not detected and repaired [4]. To cope with the high safety standards and regulations existing in highly developed industries such as aerospace, the quality of the bonded structures should be checked both after manufacturing and during usage. The lack of understanding in bonding quality with the current non-destructive testing (NDT) technologies limits adhesive bonding applications. However, it has been proved that some NDT methods can be used to detect the presence of contaminations [5-8], thus they show promises for detecting improper bonding between two items. Nevertheless, it must be noted that failures of multi-layer bonded materials can be challenging to be detected by NDT methods due to the presence of local heterogeneities along with bonded layer. 
Active Thermography (AT) is one of the most promising NDT techniques for the evaluation of the bonding quality and for determining the presence of contaminations $[9,10]$. Being a noncontact method with the capability of inspecting a large area of the Sample Under Test (SUT), AT has been extensively used for the detection of fabrication defects in bonded joints $[11,12]$. The detection and sizing of defects in adhesively bonded joints can be done by thermographic imaging, as the defect plane is typically perpendicular to the thermal wave propagation direction [13]. Surface roughness, artificial defects and grooves could be detected in adhesively bonded metallic joints using lock-in thermography [14]. The defect detection capability of traditional light-based AT techniques is highly influenced by the thermal diffusion length of the SUT. In light-based AT, the thermal waves are generated on the surface of the SUT and they need to diffuse to the required depth, being reflected by the buried defect and then back-propagate to the inspection surface to be detected by monitoring the surface temperature. To overcome this limitation, Eddy Current Pulsed Thermography (ECPT) has been recently proposed for composite evaluation. When ECPT is applied to CFRP, the stimulation can be considered volumetric since the electrical conductivity is relatively low. Depending on the excitation frequency of the Eddy Current (EC) and the sample thickness, the typical achieved skin depths are greater than the sample thickness itself, or at least comparable to it [15]. In addition, due to the multi-physics nature of the ECT, the electrical and thermal properties can be evaluated simultaneously in one experiment. ECPT is also less influenced by the surface conditions of the SUT such as emissivity and roughness [16]. Moreover, ECPT can be exploited to evaluate the barely invisible impact damage on composites $[15,17,18]$ and the presence of delamination [19-21].

However, the evaluation of the interface quality in bonded structures is still a challenging task. This is because the faithful evaluation of bonded areas is hindered by various factors: (i) their complex shape, (ii) the heterogeneous nature of the multilayer adherend, (iii) the non-uniform heating pattern combined with significant lateral thermal diffusion. All the mentioned factors contribute to the recorded thermograms and cause poor Signal-to-Noise Ratio (SNR), therefore being difficult to be interpret the results.

To tackle these challenges, a Barker Code (BC) modulated eddy current excitation combined with Pulse-compression (PuC), hereinafter called Eddy Current Pulse-compression Thermography (ECPuCT). This approach was exploited to enhance the SNR and faithfully evaluate CFRP adhesive joints containing different contaminations in the form of release agent, brass film, and release film inclusion. The system diagram is shown in Fig. 1. For each investigated sample, a series of thermograms were collected during both the heating and cooling stage shown in the first block, and the impulse response of the SUT has been derived pixelwise by using PuC algorithm presented in the second block. To find contamination layer at certain depth and conduct the characterization of different contaminations, as illustrated in the Fig. 1, three processing steps of impulse response images are undertaken: (1) non-uniform heating pattern removal by Partial Least Square (PLS) technique, as shown in the third bock; (2) time analysis carried out to select optimal time windows for Kernel Principal Component Analysis (K-PCA), shown in the third block;. (3) contamination properties comparison by proposed feature including norm of principle component, derivates of impulse response and quantification of the inclusion size by spatial kurtosis over the selected time windows.

The paper layout is as follows: Chapter II introduces the principle of ECPuCT and the mathematical description of PuC and PLS techniques. Chapter III presents the samples' manufacturing process and the experimental setup. Chapter IV gives insight about non-uniform heating removal by PLS and perfect bond subtraction, as well as the investigation of the electromagnetic behavior of contaminated bonds. Chapter V summarizes the work and explains possible future works. 


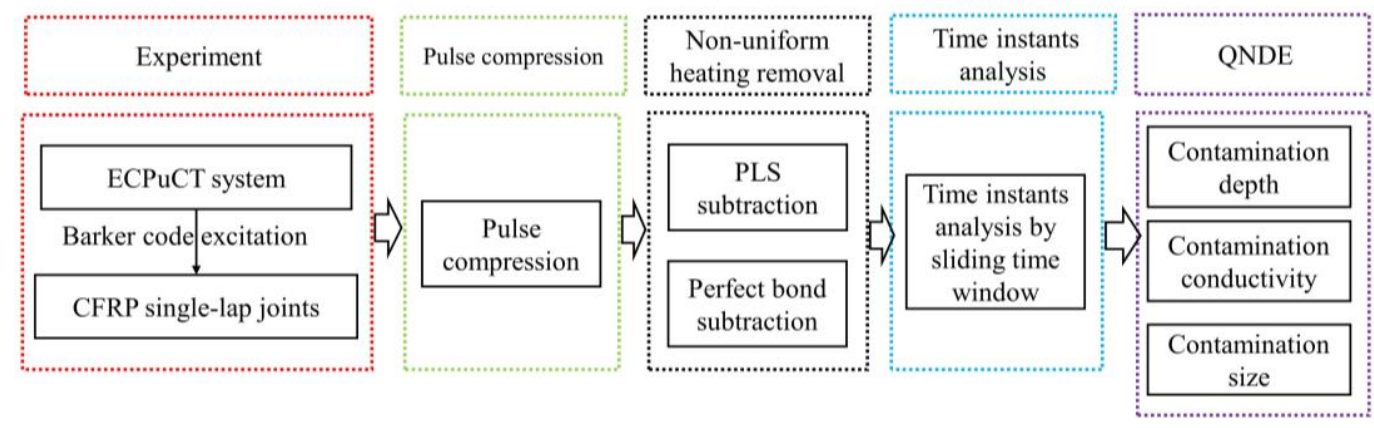

Fig. 1. System diagram

\section{Methodology of ECPuCT and feature extraction techniques}

\subsection{Principle of eddy current stimulated thermography for CFRP}

The theory of ECPT on metallic materials has been addressed in previous researches [22-24]. Even if it relies on the same physical principle, i.e. the Joule effect due to the presence of eddy current in the sample, ECPT applied to CFRP is quite different in practice for both the heating mode and the thermal wave diffusion. Indeed, the spatial distribution of eddy current into a conductive sample is regulated by the skin depth $\delta$ that decreases monotonically as the frequency increases:

$$
\delta=\frac{1}{\sqrt{\pi \mu \sigma f_{\text {carrier }}}}
$$

where $f_{\text {carrier }}$ is the frequency of the excitation current $[\mathrm{Hz}], \sigma$ is electrical conductivity $\left[\mathrm{S} \cdot \mathrm{m}^{-1}\right]$, and $\mu$ is magnetic permeability [H. $\left.\mathrm{m}^{-1}\right]$. In general, for CFRP the bulk conductivity $\sigma$ value is in the order of $15000 \mathrm{~S} \cdot \mathrm{m}^{-1}$ and it is non-magnetic [25]. Considering an $f_{\text {carrier }}=300 \mathrm{kHz}$, the corresponding penetration depth is $7.50 \mathrm{~mm}$ while for the same value of $f_{\text {carrier }}$, the penetration depth in steel $\left(\sigma=9.93 \times 10^{6} \mathrm{~S} \cdot \mathrm{m}^{-1}, \mu=3.77 \times 10^{-4} \mathrm{H} \cdot \mathrm{m}^{-1}\right)$ is equal to $29.08 \mu \mathrm{m}$, which is significantly smaller than the one obtained for CFRP. Thus, if the thickness of the CFRP sample is lower than or comparable with the corresponding value of $\delta$, as in the present case, the heating can be assumed volumetric, while in the case of steel the heating occurs in a small surface layer.

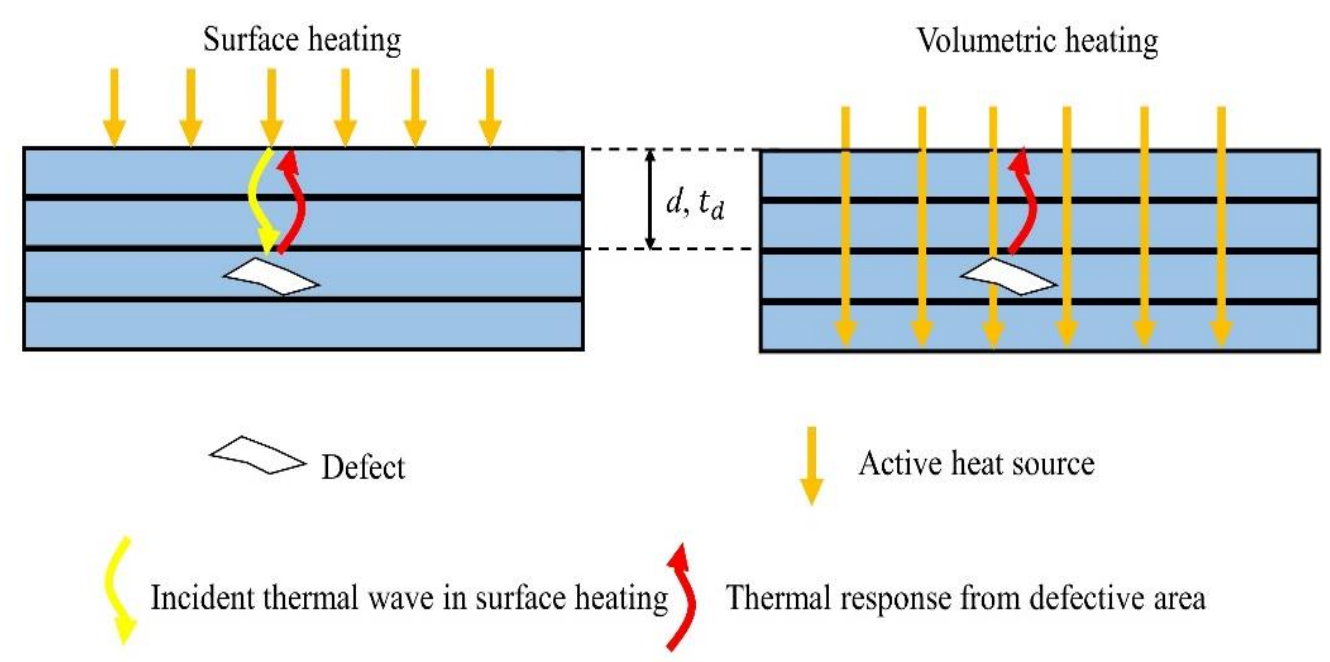

Fig. 2. Comparison of surface and volumetric heating and their effect on the detection time. 
In the case of volumetric heating, the ECPT characterization can be either in transmission or reflection mode. If the thermal diffusion length $\mu$ is greater than the depth $d$ at which the delamination/defect is possibly buried, the response from the defect can be observed through the acquired surface temperature field. The unique advantage of the volumetric heating compared to the surface heating scenarios (e.g. as in the case of light-stimulated thermography) comes from the direct interaction between the buried defect and the induced EC field. In surface heating mode, the generated thermal wave must be reflected by the defect, taking twice the time with respect to the volumetric heating mode before being recorded by the infrared (IR) camera. In addition, the thermal waves travel double the distance, which implies doubling also the attenuation in a logarithmic scale. The volumetric heating by the EC excitation is depicted in Fig. 2. The whole specimen is heated-up by the induced EC and Joule's effect [18].

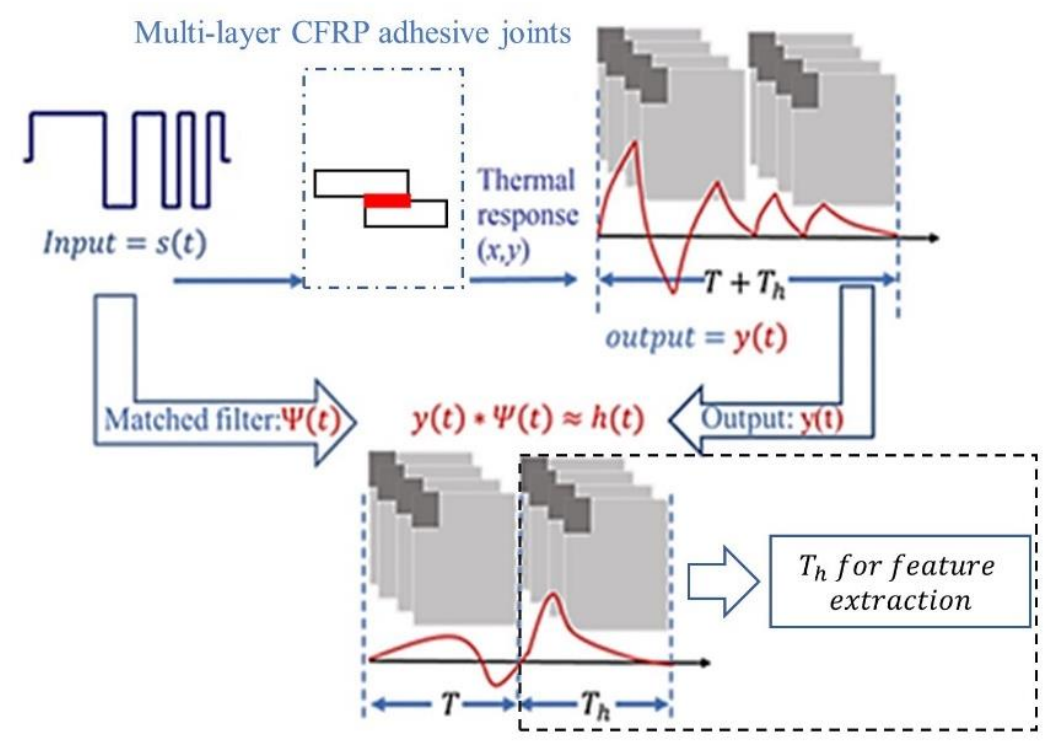

Fig. 3. Eddy current pulse compression thermography principle

The information needed for characterizing the defects comes from the thermal waves travelling from the defected area at a depth $d$ (see Fig. 2, right), which will have a different amplitude than those generated in the surrounding sound area. Instead in surface heating, the defect signal comes from the thermal waves originated at the surface and reflected by the defect. The diffusion time in this case is more than twice for the same defect depth since due to the longer distance the higher frequency components are more attenuated and the resulting mean diffusion velocity is lower.

\subsection{Pulse-compression techniques}

$\mathrm{PuC}$ is used to improve the range resolution and SNR of the signals e.g. in RADAR and ultrasonic applications. PuC has been also applied on ECPT, leading to ECPuCT [26]. In ECPuCT, the sample is excited with a coded excitation, a Barker code (BC) in the present case, of duration $T$ and thermograms are collected for an overall time duration of $T+T_{h}$. An estimated impulse response $\tilde{h}(t)$ of duration $T_{h}$ is retrieved after performing the $\mathrm{PuC}$ algorithm. PuC relies on the existence of a pair of signals: an excitation signal $s(t)$ of duration $T$ and bandwidth $B$, and a matched filter $\Psi(t)$, such that their convolution $s(\mathrm{t}) * \Psi(\mathrm{t})$ approximates the Dirac's Delta function $\delta(t)$ :

$$
s(t) * \Psi(t)=\tilde{\delta}(t) \approx \delta(t) .
$$

If Eq. (2) is satisfied, and $s(t)$ excites a SUT that is characterized by the ideal impulse response $h(t)$, then an estimation of the $h(t)$, i.e. $\tilde{h}(t)$, can be obtained by convolving the output signal $y(t)$ with $\Psi(t)$. Eq. (3) shows the mathematical formulation for a single pixel of the acquired 
thermograms, in presence of an Additive-White-Gaussian-Noise $e(t)$, which is considered uncorrelated with $\Psi(t)$. The estimated impulse response can be obtained as:

$$
\begin{gathered}
\tilde{h}(t)=y(t) * \Psi(t) \stackrel{y(t)=h(t) * s(t)+e(t)}{\stackrel{(}{\longrightarrow}} h(t) * S(t) * \Psi(t)+e(t) * \Psi(t)= \\
=h(t) * \tilde{\delta}(t)+\tilde{e}(t) \approx h(t)+\tilde{e}(t) .
\end{gathered}
$$

The main advantage of ECPuCT compared with ECPT is that the impulse response can be estimated by delivering energy to the system in a significantly longer time. This flexibility can be exploited, as in the present case, either to increase the SNR by providing more energy than in the ECPT scheme or to use lower power heating sources while maintaining the same SNR level. In this paper, $s(t)$ is a $\mathrm{BC}$ of order equal to 13 and the matched filter $\Psi(\mathrm{t})$ is the time-reversed sequence of the input coded signal, i.e. $s(-t)[27]$.

\section{$2.3 \quad$ Non-uniform heating removal by Partial Least Square}

As shown in the block 3 of Fig. 1, to remove the non-uniform heating pattern, the PLS technique was applied to reconstruct the non-uniform heating pattern generated by the coil shape and the anisotropy of the material properties. As for the implementation, the PLS can reconstruct the whole three-dimensional data $(x, y, t)$ to characterize the non-uniform heating pattern at each time. Thus, by subtracting the original data by the PLS reconstructed data, the surface condition and anisotropic conductivity influences are mitigated. The algorithm is depicted in Fig 4 and mathematical formulation is shown below.

The set of impulse responses $H_{N_{x} \times N_{y} \times N_{t}}$ retrieved after PuC is reshaped from $3 \mathrm{D}$ to $2 \mathrm{D}$ as $H_{N_{t} \times\left(N_{x} * N_{y}\right)}$, which can be shortened as $H_{\text {set }}$. The PLS mathematical modelling of $H_{\text {set }}$ is conducted as:

$$
H_{\text {set }}=T P^{T}+E, \quad Y=T Q^{T}+F,
$$

where $Y_{N_{t} \times 1}$ is corresponding to each frame number vector $\left[1, \ldots, N_{t}\right]$ and $T_{N_{t} \times a}$ is the score matrix. The scores are interpreted as latent variables of each frame provoking systematic variation in $H_{\text {set }}$. $P_{N_{t} \times a}$ and $Q_{a \times 1}$ are the loadings matrices, which describe the way of projecting $T_{N_{t} \times a}$ to $H_{s e t}$ and $Y$ respectively. $H_{\text {set }}$ and $Y$ are assumed to be partly modelled by same latent variables $T_{N_{t} \times a}$. The score matrix $T_{N_{t} \times a}$ columns are orthogonal to each other and $H_{\text {set }}$ can be estimated as linear combination of $T_{N_{t} \times a}$ with coefficient weights $W_{\left(N_{x} * N_{y}\right) \times a}$. Thus, scores $T$ can be expressed as:

$$
T=H_{\text {set }} W \text {. }
$$

After the calculation of scores, loadings $P$ and $Q$ are estimated through the regression of $H_{\text {set }}$ and $Y$. Then, residual matrices are computed by subtracting the estimated $T P^{T}$ and $T Q^{T}$ from $H_{s e t}$ and $Y$, respectively. The regression coefficients $B$ for the model are obtained using the following equations:

that can be solved as:

$$
B=W Q^{T},
$$

$$
Y=H_{\text {set }} B+F=H_{\text {set }} W Q^{T}+F .
$$

The PLS problem is solved based on Iterative Partial Least Square (IPALS) algorithm. After obtaining PLS components $T P^{T}$ of the data $H_{\text {set }}$, the first PLS component is applied to reconstruct the non-uniform heating pattern and PLS subtraction is conducted on the first component reconstructed data. 


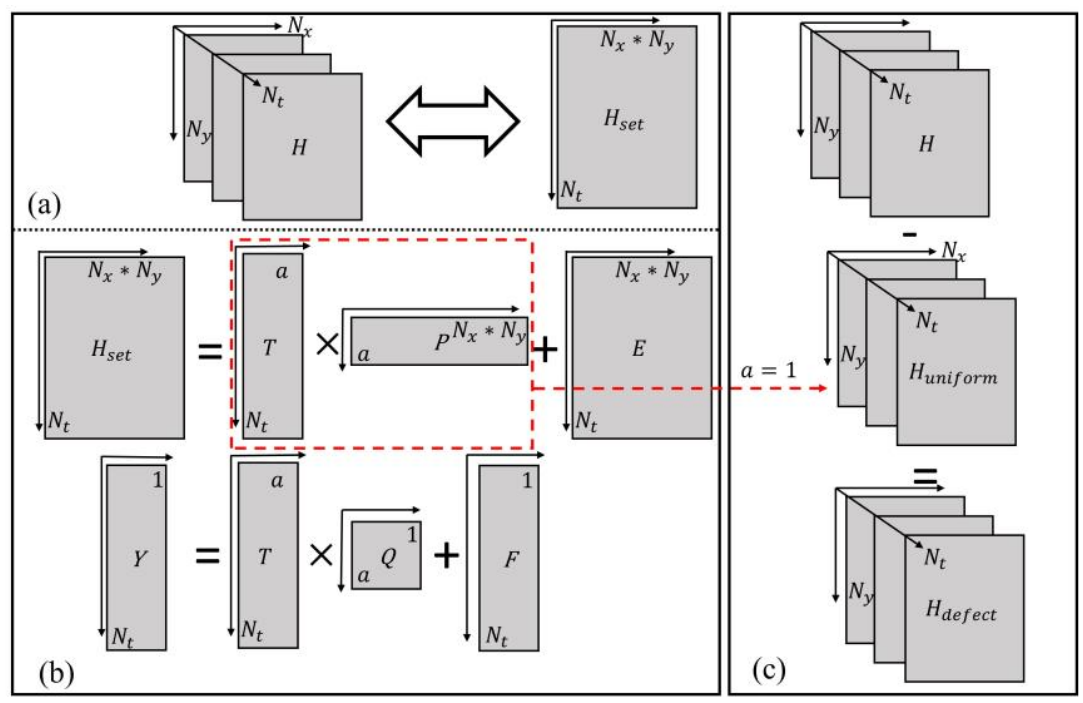

Fig. 4. Implementation process for PLS subtraction: (a) Data reshape process, (b) PLS component calculation, (c) Subtraction on the first PLS component

\subsection{Feature extraction techniques for impulse response}

This section introduces the approach to locate the time when the information is maximum about the contamination layer. The evaluation of contamination properties is conducted based on optimal time window. The process to evaluate the electrical and thermal properties of the contamination is based on the selection of a proper time window within the pixelwise-retrieved $\tilde{h}(t)$ that provides the information about the contamination layer. The proposed procedure is schematically depicted in Fig. 5.

If $H_{N_{x} \times N_{y} \times N_{t}}$ is segmented evenly in time as $h_{i, i+1}$, where $i$ denotes the time position of a chosen time window, then the projected image can be calculated as $\alpha_{i 1} * h_{i, i+1}{ }^{T}$ by using K-PCA for $h_{i, i+1}$ to obtain the first principal component. For each projected image $\alpha_{i 1} * h_{i, i+1}{ }^{T}$, the contrast of the delamination over sound area is considered as the norm of each corresponding $\alpha_{i 1}$, which is presented as the Sliding K-PCA part in Fig. 5. By selecting the best image with positive local maximum $\alpha_{k 1}$, the corresponding image is reconstructed as $\alpha_{k 1} * h_{k, k+1}{ }^{T}$. To understand if the period $\alpha_{k 1} * h_{k, k+1}{ }^{T}$ contains the maximum information, derivate and time-phase of the impulse responses are used, as shown in the middle part of Fig. 5. Then, the image $\alpha_{k 1} * h_{k, k+1}{ }^{T}$ is used to locate the contamination response $h_{k, k+1}$ for brass film, release film, and release agent, as it is shown in Fig.5 (QNDE labelled part). The contamination properties are characterized by the norm distance of $\alpha_{k 1}$, and the size of brass film and release film is determined by spatial kurtosis on the image $\alpha_{k 1} * h_{k, k+1}{ }^{T}$. 


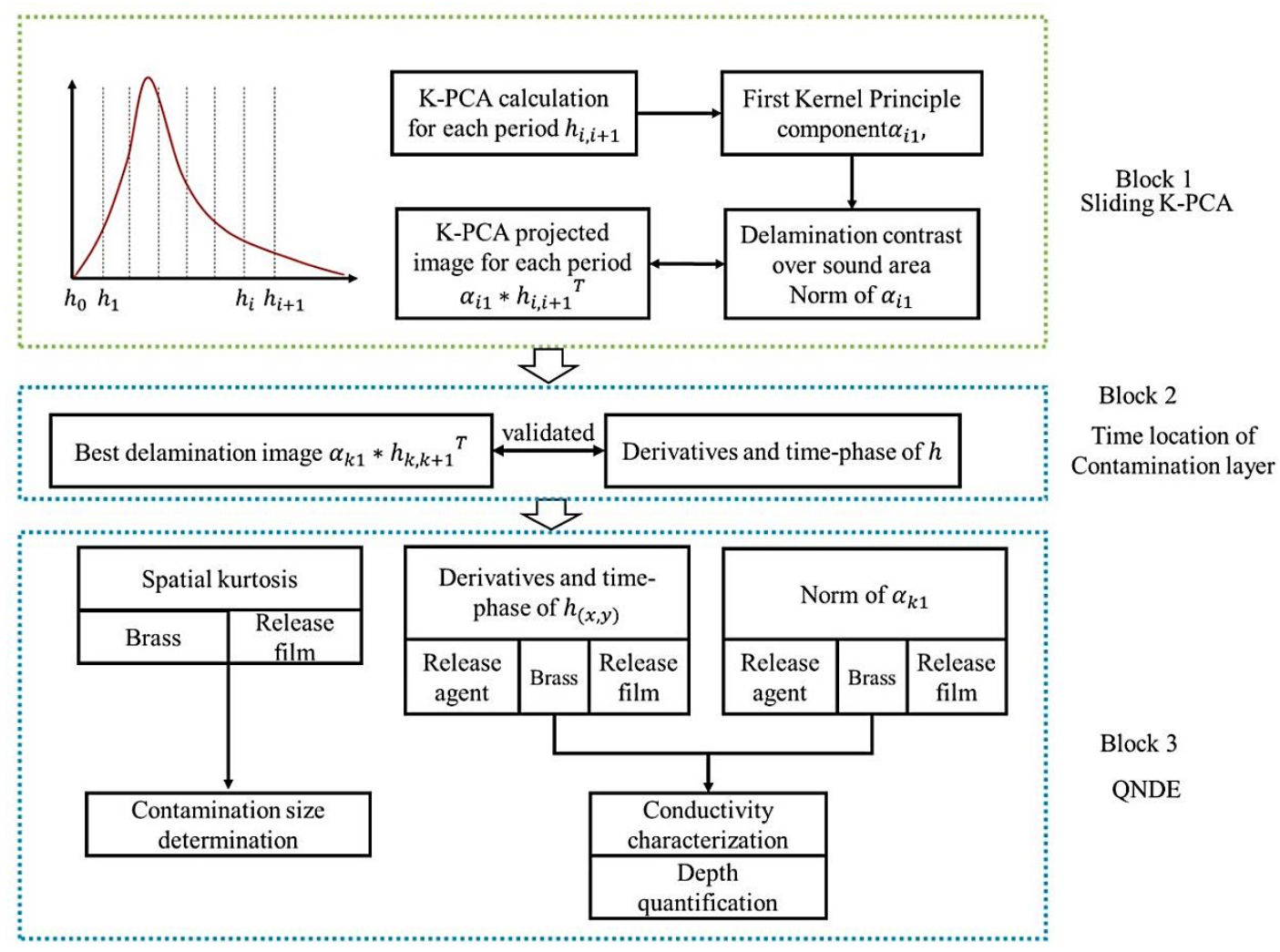

Fig. 5. Diagram depicting the proposed methodology for the characterization of contamination properties at the bonding interface.

\section{Experimental setup and the sample under test}

ECPuCT system setup is introduced in the Section 3.1 and CFRP single lap joints with different contaminations including highly electrically conductive brass, release film and release agent with low electrical conductivity are introduced in Section 3.2.

\subsection{ECPUCT system}

The ECPuCT system diagram is depicted in Fig. 6(a). A signal generator was used to send both the $\mathrm{BC}$ modulating signal to the induction heating unit and a reference clock trigger to the IR camera to acquire thermograms at 50 Frames Per Second (FPS). A Cheltenham EasyHeat 224 induction heating unit was employed for exciting the coil with a maximum excitation power and current values of $2.40 \mathrm{~kW}$ and 400 A respectively, with tunable $f_{\text {carrier }}$ within 150 to $400 \mathrm{kHz}$. For the reported experimental results, values of excitation current $I$ equal to $40 \mathrm{~A}$ and $f_{\text {carrier }}$ of $240 \mathrm{kHz}$ were selected to prevent damaging the CFRP sample due to the long excitation time using $\mathrm{BC}$ signal.

Fig. 6(b) shows a picture of the employed rectangular coil made from high conductivity hollow copper tube with the outer and inner diameters of $6.35 \mathrm{~mm}$ and $4.00 \mathrm{~mm}$ respectively. Water cooling was implemented to the coil to avoid over-heating and the lift-off maintained at $3.00 \mathrm{~mm}$ from the SUT's surface to ensure the volumetric heating of the sample. Only one side of the coil is selected as a linear coil to induce parallel eddy currents in the direction of maximum conductivity within the sample, the length of this side was $9.30 \mathrm{~mm}$. The IR camera was the FLIR SC655, equipped with an un-cooled microbolometer detector array having a resolution of $640 \times 480$ pixels, spectral range of $7.5-14.0 \mu \mathrm{m}$ and NETD $<30 \mathrm{mK}$. The IR camera recorded the SUT's surface temperature distribution during the whole $13 \mathrm{~s} \mathrm{BC}$ modulated stimulus, as well as for an additional $30 \mathrm{~s}$ of cooling period [28] with a frame rate of $50 \mathrm{~Hz}$, i.e. 50 FPS. Please note that the 13 seconds of $\mathrm{BC}$ and the additional 30 seconds of acquisition correspond to $T+T_{h}$ described in Section 2.2. 
Finally, the captured thermogram sequences were transmitted to a PC for visualization and postprocessing, including signal pre-process, $\mathrm{PuC}$, thermal pattern enhancement and feature extraction.

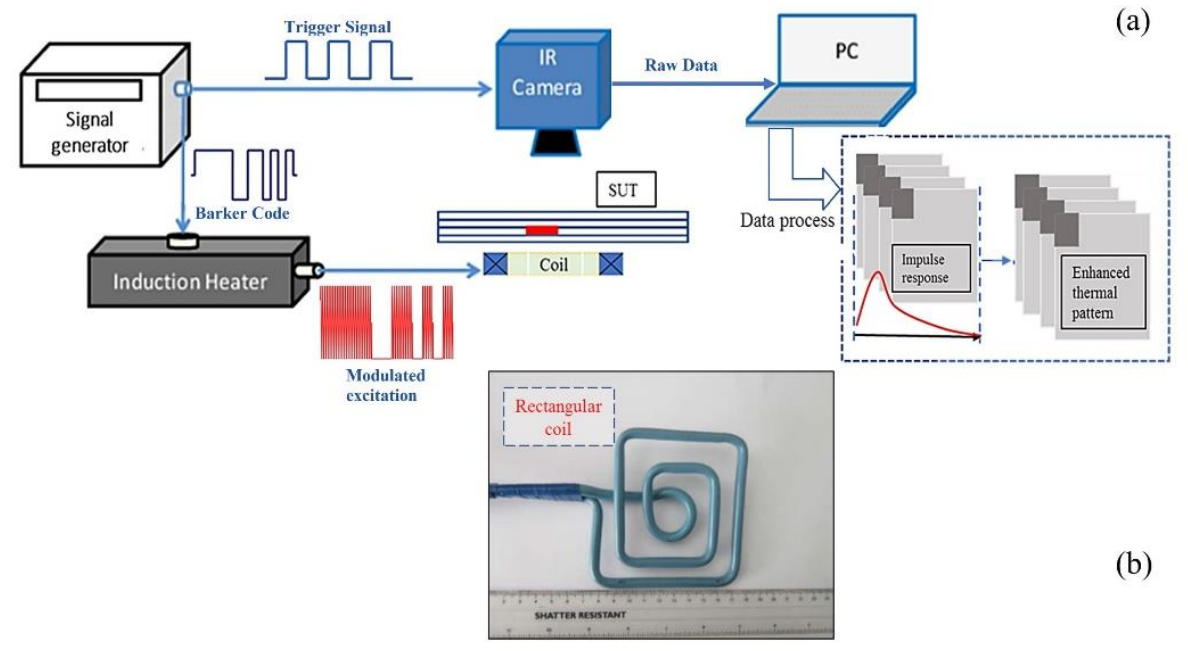

Fig. 6. Experimental setup: (a) system block diagram, (b) detail of the employed rectangular coil

\subsection{Investigated Samples description}

As explained in Section 1, three types of contaminated debonding of brass, release film and release agent were manufactured. The composite plates were manufactured using six plies of HexPly M21 - 5H Satin woven prepreg. Due to woven structure and the number of lay-up, residual stress can occur in specimens. The thickness of the specimens may vary through the structure. However, the mentioned variation is here negligible as it is in the range of $10^{-6} \mathrm{~m}$. The interfacial contaminations were realized by using brass film, Wrightlon 4600 release film and Marbocote 45 release agent. As it is shown in Fig. 8(a), two CFRP plates were overlapped to create a single lap joint, such that the bonded area was $280 \mathrm{~mm} \times 25 \mathrm{~mm}$ while the total area of each composite plate was $280 \mathrm{~mm} \times 120 \mathrm{~mm}$

In the bonding area, two-fold brass film and release film inserts were added to simulate debonding at the interface; release agent was sprayed over the epoxy film sheet prior to second adherend placement in order to weaken the epoxy quality. As shown in Fig. 7(b) and Fig. 8(b), five square release films were placed at the upper interface, having a nominal thickness of $0.076 \mathrm{~mm}$ and size of $12.70 \mathrm{~mm} \times 12.70 \mathrm{~mm}$. Ten square-shape brass inclusions all having thickness equal to $0.05 \mathrm{~mm}$ were embedded into the sample as depicted in Fig. 7(a), Fig. 8(c). Please note that these brass inclusions had different sizes: bigger ones are $12.70 \mathrm{~mm} \times 12.70 \mathrm{~mm}$, and smaller ones $6.35 \mathrm{~mm} \times 6.35 \mathrm{~mm}$. As for the weak bond sample, the part with less contamination was made by spraying the release agent on a mask above the uncured adhesive epoxy, as shown in Fig. $7(\mathrm{c})$. On the other hand, the part with more contamination was made by spraying the release agent uniformly on the whole bonding area, as shown by a blue area in Fig. 7(c). It should be noted that in latter case, the release agent has been diffused into the uncured epoxy, which may result in untraceable amounts at the interface between epoxy adhesive and composite adherend. 

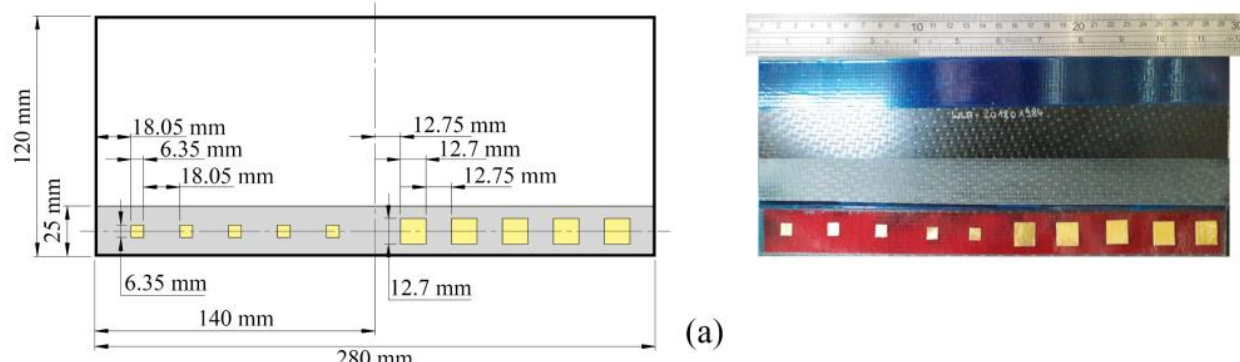

(a)
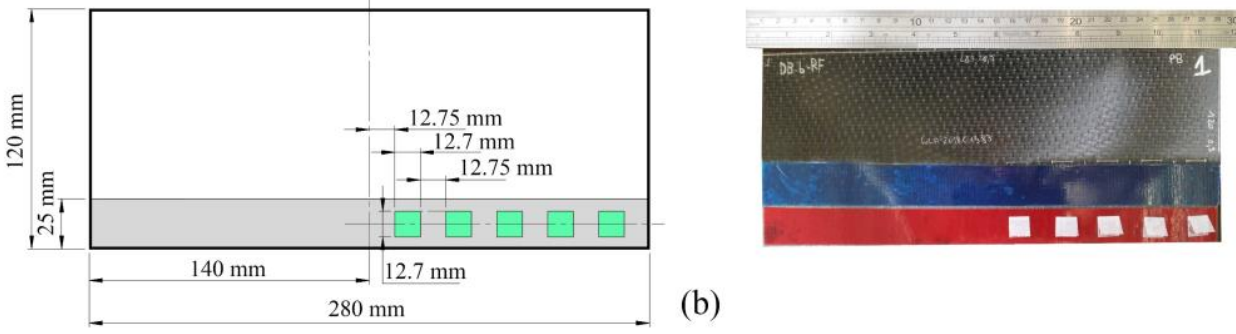

(b)
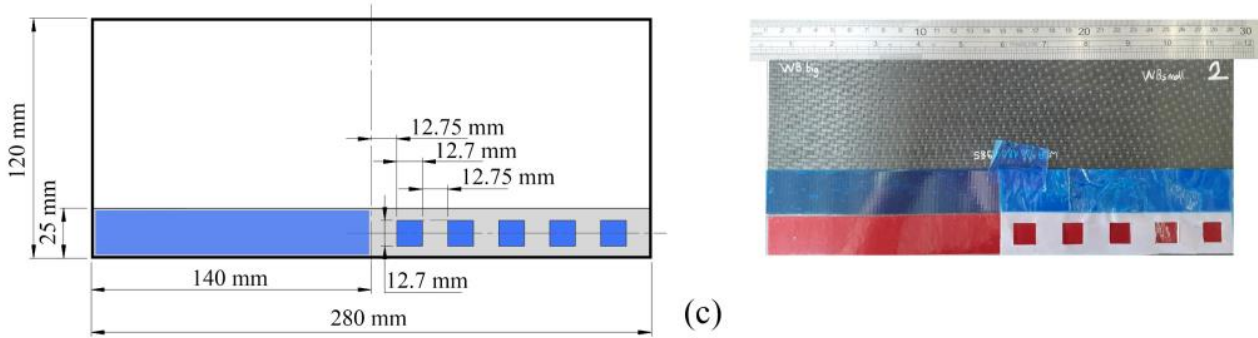

(c)

Fig.7. CFRP-epoxy adhesive single-lap joints plan sketch: (a) Perfect bond and release film inclusion, (b) Brass film inclusion, (c) Release agent inclusion

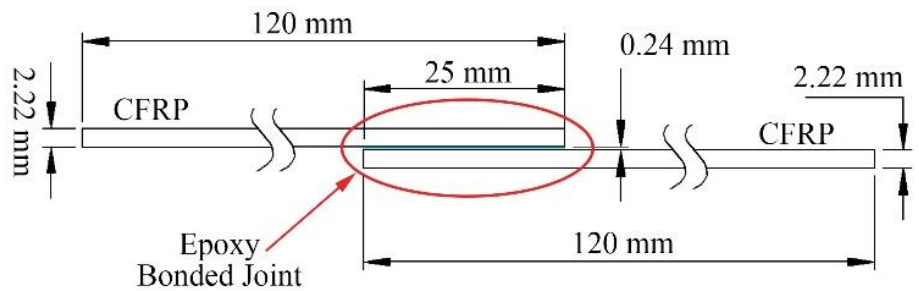

(a)

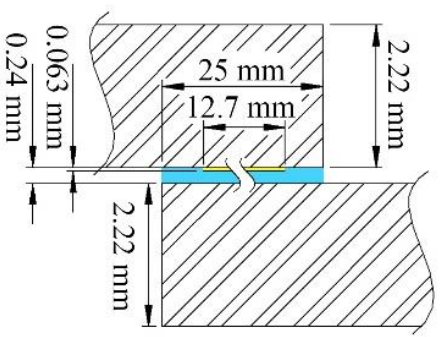

Release film inclusion

(b)

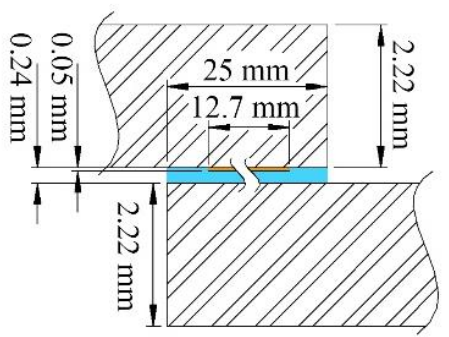

Bigger brass inclusion

(c)

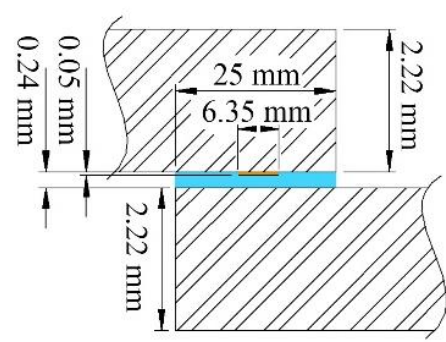

Smaller brass inclusion

(d)

Fig. 8. CFRP-epoxy adhesive joints cross section sketch: (a) Perfect bond, (b) Debonding with release film, (c) Debonding with bigger brass film, (d) Debonding with smaller brass film, (e)

Weak bond with less contamination. (f) Weak bond with more contamination 


\section{Results and discussion}

In this section, the PuC thermal data are pre-processed to remove non-uniform heating by PLS, time window sliding for depth detection of contamination and quantitative evaluation of contamination layers including classification and sizing of contamination as illustrated in Fig. 1, QNDE part.

\subsection{Non-uniform heating pattern removal}

The non-uniform heating pattern usually generated by coil in induction heating can hinder the ability to detect and locate the defect. Thus, as it is shown in third block of Fig 1, this section focusses on the implementation of non-uniform heating pattern removal. The non-uniform heating pattern generated by the anisotropy of the material, geometry and coil shape should be removed to obtain a more uniform image. This would help achieving a better feature extraction. Two different methods have been used to remove non-uniform heating pattern: PLS and perfect bond subtraction using a reference.

In this work, PLS reconstructed thermal data is subtracted from the PuC thermal data. As shown in Fig. 4, only the first PLS component is used to reconstruct the non-uniform heating because according to [29], the first component contains the heating pattern, while second or third components might carry defect information. Besides the PLS approach, repeatable experiment on the perfect bonding sample is also conducted to obtain data without defect but containing nonuniform heating pattern. The results of both approaches are illustrated in Fig 9. In Fig. 9(a), the $12.70 \mathrm{~mm} \times 12.70 \mathrm{~mm}$ brass film is identified by three heated fibers shown in K-PCA1 using perfect bond and PLS subtraction methods respectively. It is of utmost importance to note that the PLS subtraction method does not require to gather data from the sound sample, thus the misalignment of spatial coordinates caused by different experiments is avoided. Instead, further efforts are needed to subtract perfect bonding in a proper way. Therefore, in the case of K-PCA1 with PLS subtraction, the brass pattern is more uniform and closer to the real square shape than the one obtained over the same sample using perfect bond subtraction (see third row of Fig. 9(a)). However, the pattern of the smaller brass film inclusion $6.35 \mathrm{~mm} \times 6.35 \mathrm{~mm}$ is not clearly visible in Fig. 9(a) that can be due to the texture of the fibers above the brass. In the case of release films depicted in Fig. 9(b), the defects are more clearly visible. The difference in the SNR of the patterns from bigger brass and the release film is due to the fact that brass inserts have higher electrical conductivity than the sound sample, hence not only allowing eddy currents flowing but also heating up due to the Joule's effect. Release film defects are not electrically conductive, therefore completely interrupt and deviate the eddy current flux around them.

It can be observed in the first row of Fig. 9 that the K-PCA1 obtained from the raw acquired $h(t)$ 's contains the elliptical thermal pattern generated by the excitation coil. On the other hand, by subtracting either the perfect bond or the PLS data from the various contaminated bonding data, the debonding area is further enhanced, as it is visible in second and third rows of Fig. 9. For the release agent inclusion, the detection capability is heavily reduced as shown in Fig. 9(c) and (d). The contrast between more release agent inclusion and sound area is observable in Fig. 9(c). The release agent might have been diffused into the epoxy resin or distributed uniformly along the bonded area. However, in Fig. 9(d), the defect pattern is similar to the non-uniform heating pattern, which shows that less release contamination agent is not detectable. 
Based on the discussion above, the proposed non-uniform heating removal process using PLS techniques can remove the non-uniform heating pattern for detection of contamination. Mismatch of spatial coordinates can be avoided through PLS subtraction.
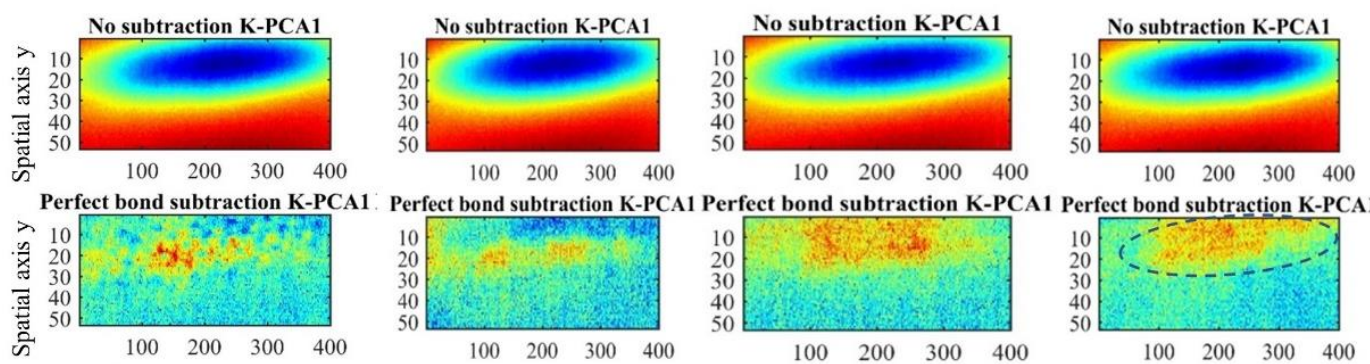

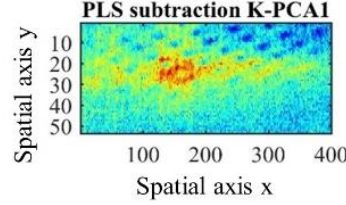

(a)

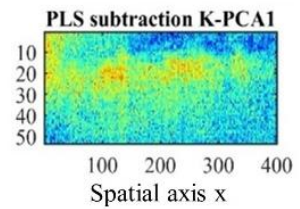

(b)

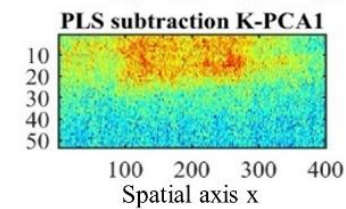

(c)

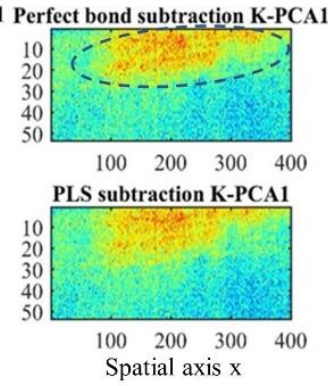

(d)

Fig. 9. Results obtained without subtraction and with subtraction of PLS and perfect bond for: (a)

Brass film, (b) Release film, (c) More release agent contamination, (d) Less release agent contamination

\subsection{Multi-physics analysis based on sliding window of $K-P C A$}

In order to find the time instance carrying the maximum information about the contamination layer, physical analysis of the eddy current and thermal phenomena based on sliding K-PCA is conducted. The impulse response-based K-PCA calculation is presented in previous work [26]. The time series of the images is subdivided into non-overlapping time-windows each one having five frames. In K-PCA, the linear kernel is applied due to the limited number of data in five frames. The calculation is based on the first principal component, which can help compress major information. The physical meaning of K-PCA1 after PLS subtraction is to observe the variations of SUT's thermal response. The feature used as depth indicator is the norm of first principal component for every five frames within the first 80 frames of PLS subtracted data.
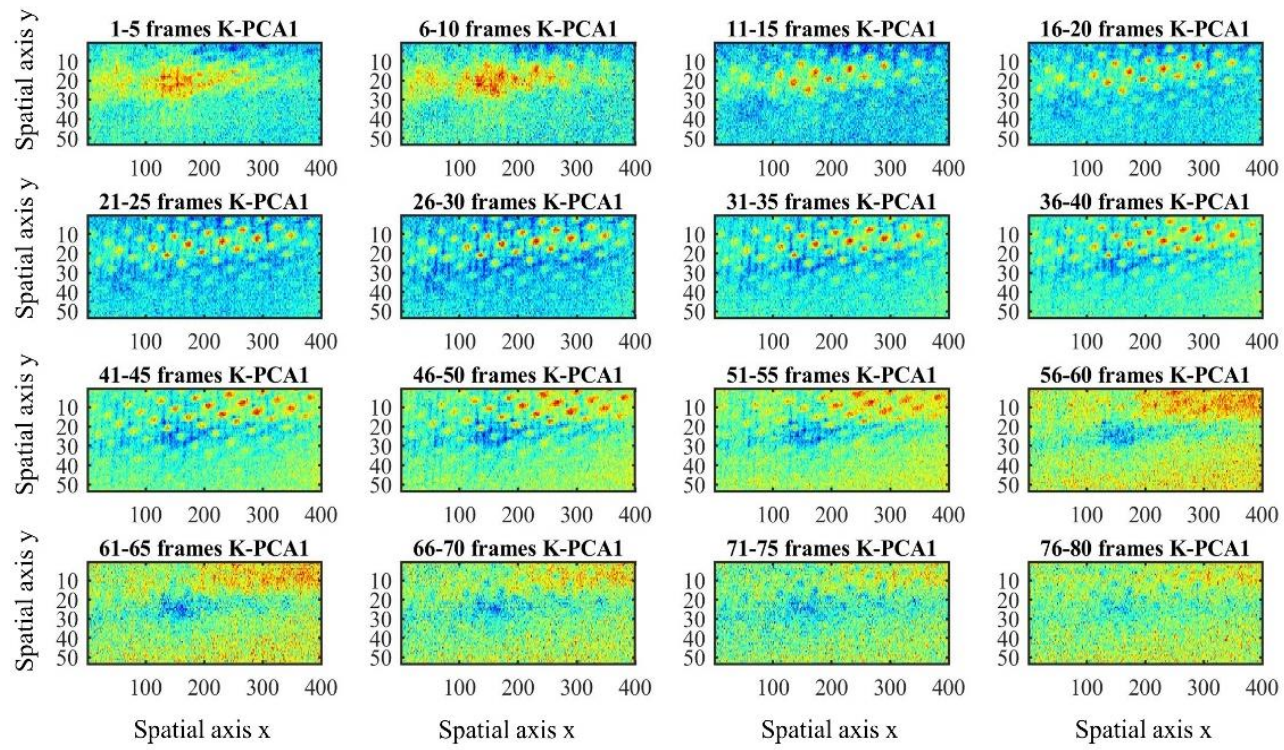

Fig. 10 Sliding K-PCA results for brass film inclusion 

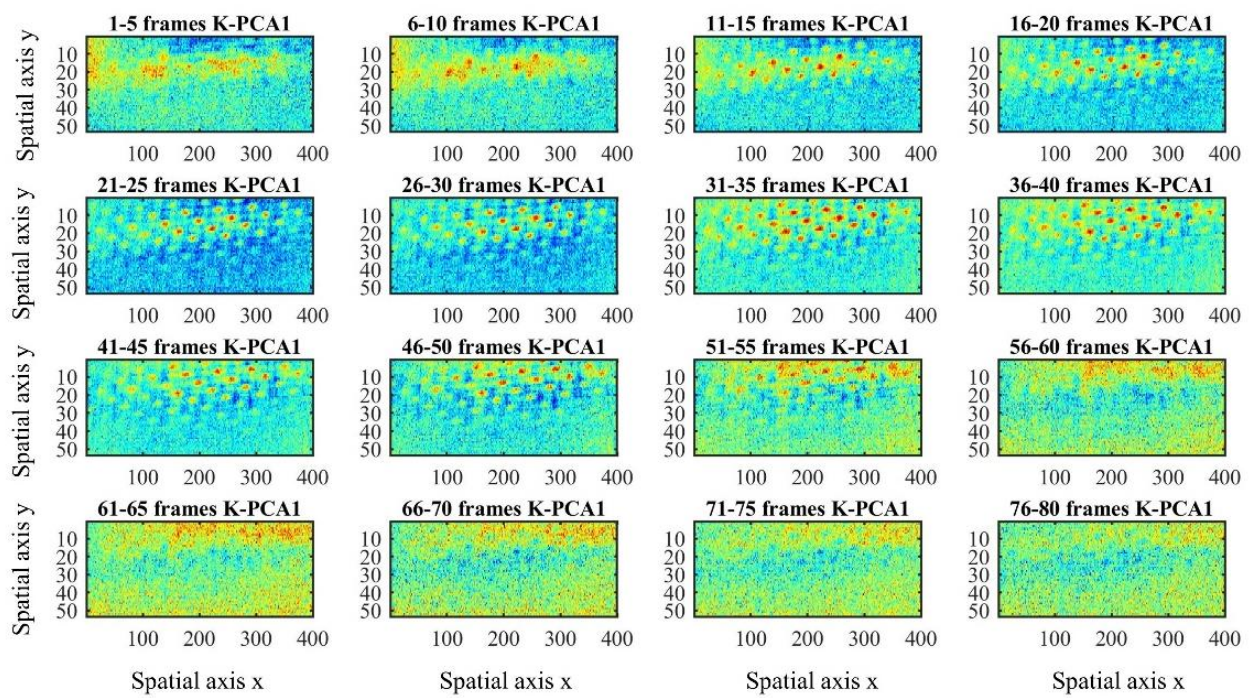

Fig. 11 Sliding K-PCA results for release film inclusion

The Sliding K-PCA results of brass, release film and release agent inclusions are presented in Fig. 10 to Fig. 12 for every 5 frames. As shown in Fig. 10, a higher contrast between brass and sound area is achieved in the first ten frames. This can be explained by the higher electrical conductivity of brass, so that larger eddy current density is expected than that in the surrounding CFRP and bonding material. According to [30], in the first phase of the induction heating EC is the dominant effect, thus the higher the conductivity, the higher the EC density and the resulting heating due to the Joule's effect. As shown in Fig. 10 and Fig. 11, the highest contrast is also found at the beginning for release film and release agent samples. The difference between conductive contamination (brass) and non-conductive ones (release film and release agent) is the time to reach the peak contrast. It can be seen in Fig. 13 that it only takes 5 frames $(100 \mathrm{~ms})$ for the brass to reach its maximum contrast but for non-conductive contaminations it takes 10 frames (200 ms). In Fig. 10 to Fig. 12, from frame 11 to 25 , the defect pattern is covered by the response from the texture of the CFRP layer over the debonding area. Heat diffusion between the layers plays a major role in the detection of debonding: for brass film, the heat diffuses from brass to the surrounding, for release agent contamination or release film, the heat from the surrounding CFRP diffuses into the contaminated area.
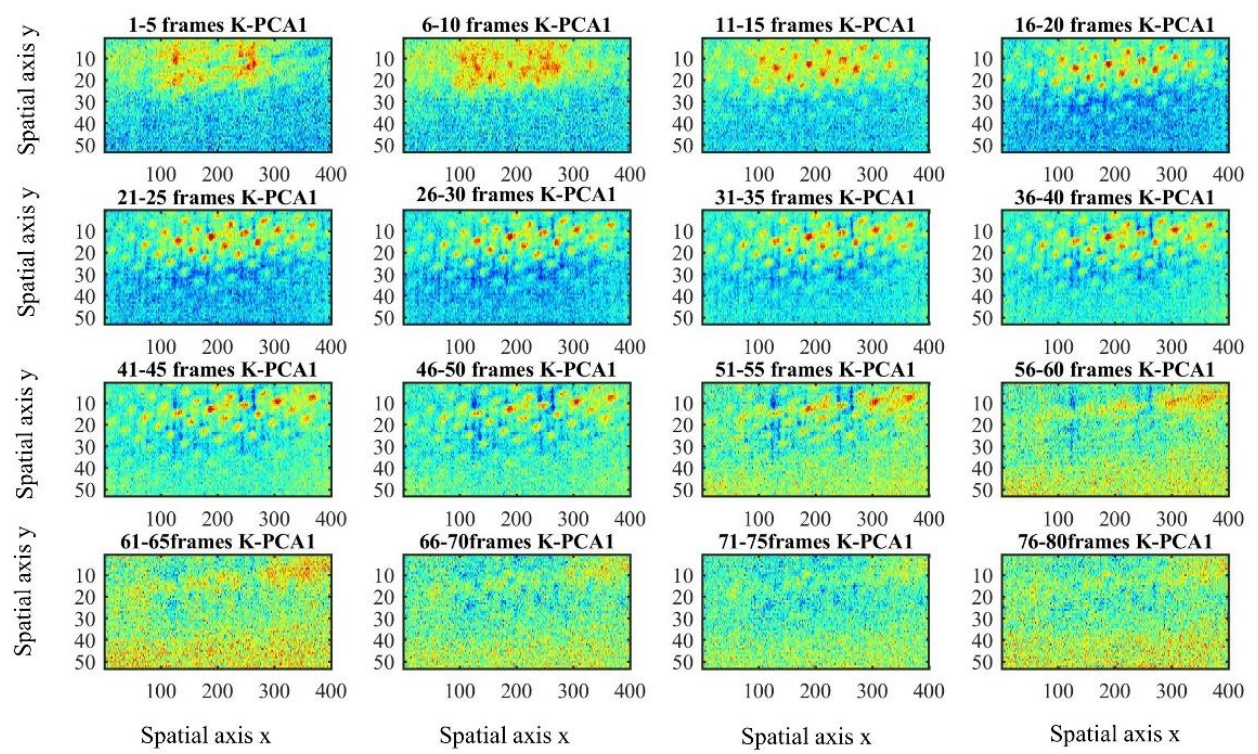

Fig. 12. Sliding K-PCA results for weak bond with more contamination 
Within the 80 frames, shown in Fig. 13, first 15 frames indicate the maximum domination of the Joule's effect, while in the cooling stage, around frame number 60 , thermal diffusion plays a significant role.

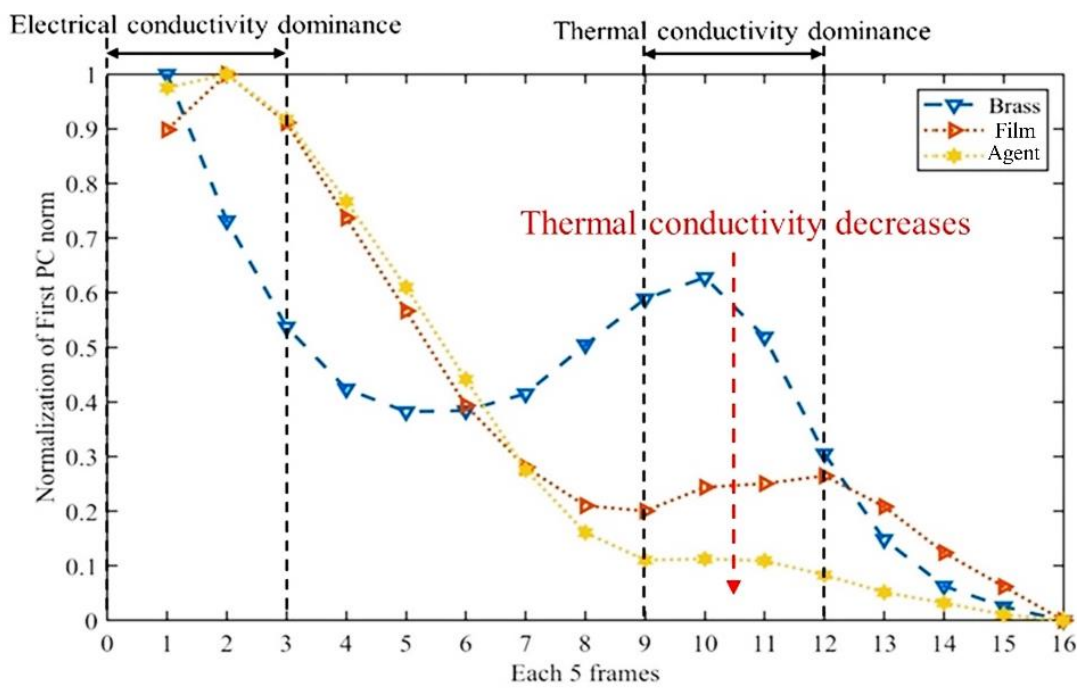

Fig. 13. First PC norm of different contaminations versus number of frames

\subsection{Classification of contamination and Quantitative evaluation of size}

In this Section, the impulse response and its derivatives are compared to classify thermal and electrical properties of contamination materials as well as the brass size. In Fig. 14(a), it can be observed that at the beginning of the heating stage, the impulse response of brass rises quicker than other two contaminations and CFRP due to its higher electrical conductivity. The release film shows the least significant response to the Joule's effect because it is not conductive. Impulse response of intact CFRP shows a slightly higher heating response than the release agent at its peak time.

It is observed that the peak value and its time instant for the brass is different from release film inclusion, which has a later peak time than the CFRP and release agent as shown in Fig 14(c). After reaching the peak, the release film inclusion cools down slower than both intact CFRP and weak bond as it hampers the heat diffusion. This effect is visible in Fig. 14(a).

However, it is challenging to evaluate the thermal conductivity of the brass contamination only by the impulse response because the Joule heating is more significant in the brass with respect to the sound CFRP surrounding area. Therefore, the heat diffuses from the brass to the surrounding area even at the cooling stage. Thus, the derivative of the impulse response can be used to evaluate the thermal conductivity as shown Fig. 14 (b). Fig. 13 and Fig. 14(b) show similar trends, i.e. electrical conductivity plays a dominant role at around 10th frame while thermal conductivity shows a prominent role at 60th frame. It can be seen in Fig. 14(b) that at 60th frame the absolute value of impulse response's derivative from brass has higher value than other contaminations, proving its higher thermal conductivity. Comparing the difference of release agent and CFRP in heating and cooling stages in Fig. 14(b), it is observed that the release agent has more influence on thermal conductivity of contaminated area than the electrical conductivity. In addition, by using the time-phase information of the impulse responses defined through Hilbert transform [31], it can be observed that the phase turning point also happens at $60^{\text {th }}$ frame (see Fig. 14(d)). Please note that the turning point happens at the same value at which there is the transition in Fig. 13 and Fig. 14(b). 


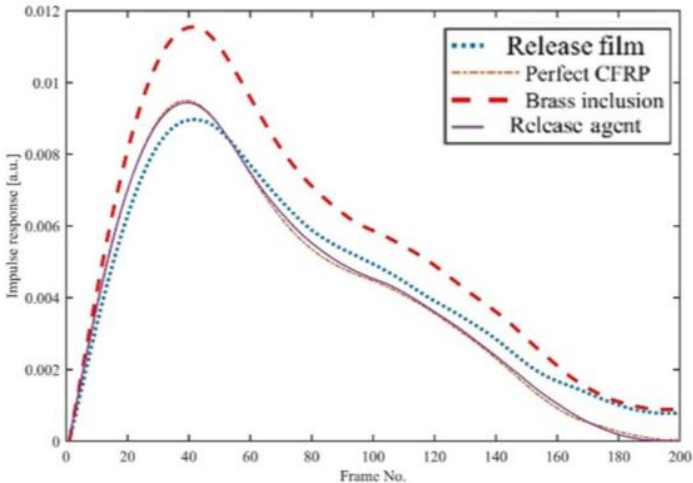

(a)

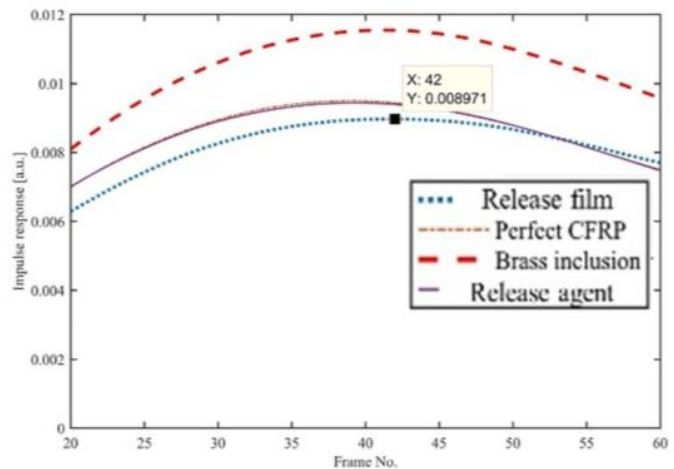

(c)

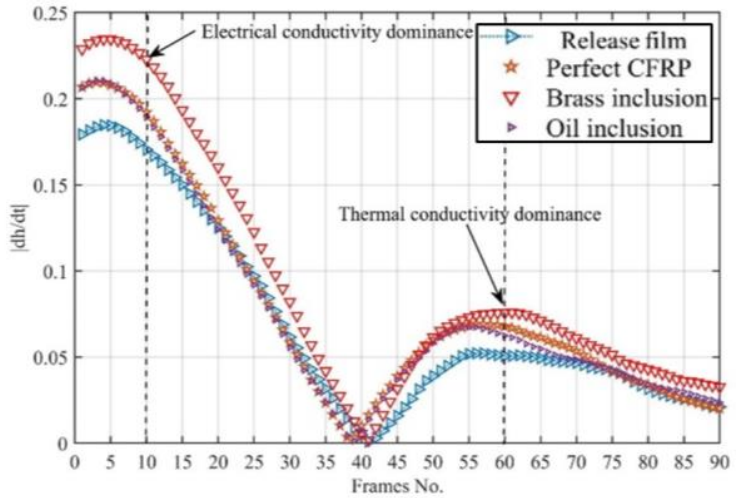

(b)

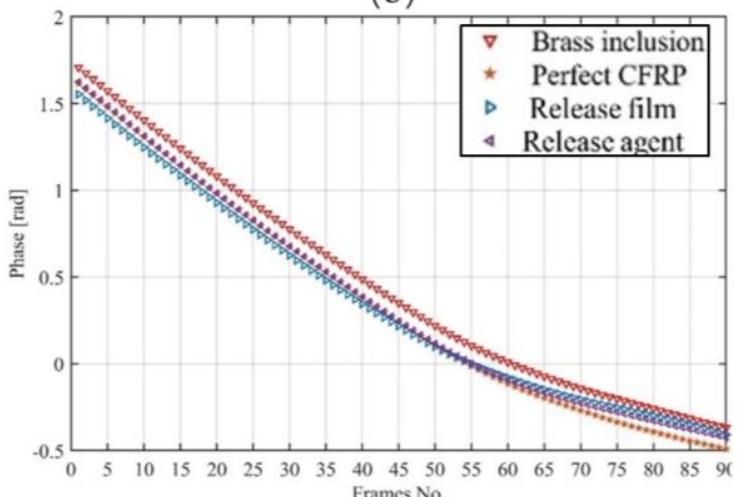

(d)

Fig. 14. Data of different material at selected position (a) Impulse responses, (b) Absolute value of impulse response derivatives, (a) Zoom of the peak value of impulse responses, (d) Timephase variations of different inclusions

In order to determine the size of contaminations, spatial kurtosis is applied to the data. The sizing data was derived from five pixels lines of data in the best contrast image. Since the size of bigger brass is $12.70 \mathrm{~mm} \times 12.70 \mathrm{~mm}$ and smaller one is has one fourth of the area, it is observed that the spatial kurtosis of smaller one over bigger one is $2.05 / 3.30=0.62$, which is slightly bigger than 0.5 for the width ratio shown in Fig. 15. This can be caused by the texture influence, which has been explained in Section 4.1. For the release films, the kurtosis ratio of two same films with the same size is 3.11/2.95=1.05, which indicates their similar size as shown in Fig. 16.

It is proved that at first 15 and 60 frames the physical properties of inclusions can be classified and using the spatial kurtosis at $15^{\text {th }}$ frame brass size can be quantified.

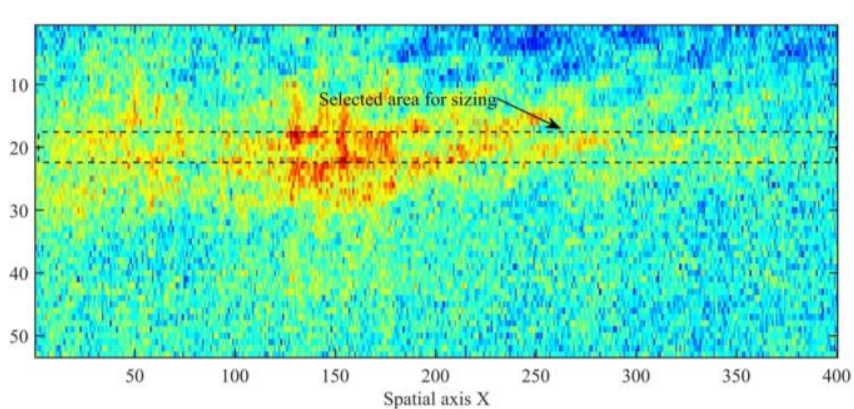

(a)

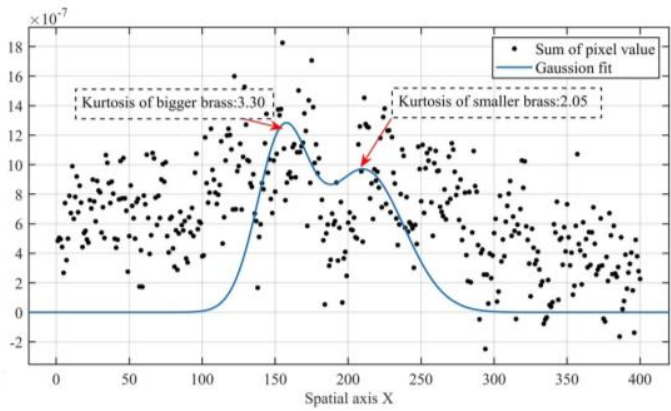

(b)

Fig. 15. Spatial kurtosis for sizing of bigger and smaller brass films: (a) Selected area for size quantification, (b) Results of spatial kurtosis 


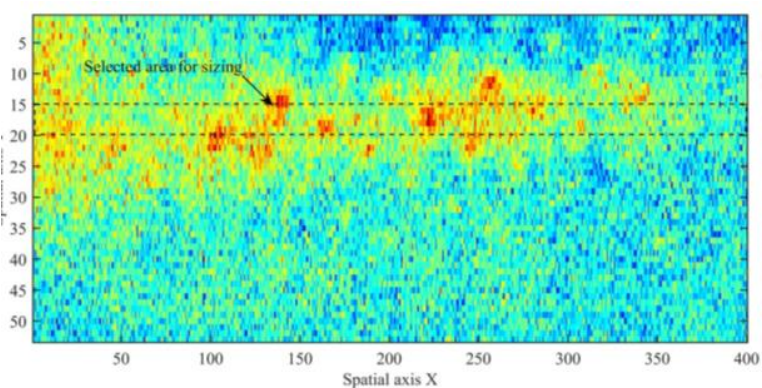

(a)

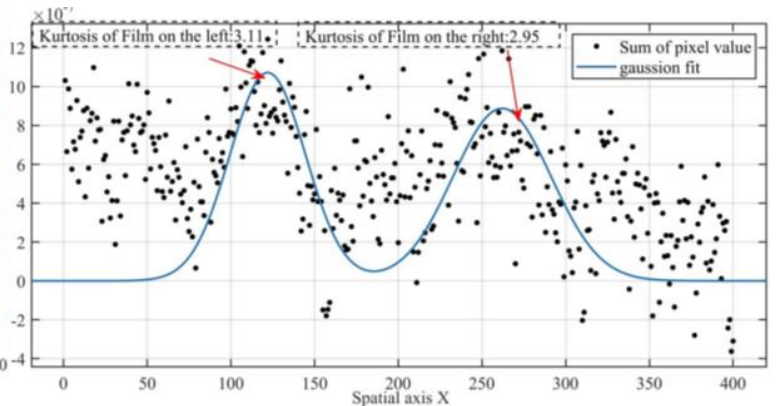

(b)

Fig. 16. Spatial kurtosis for sizing of the release films. (a) Selected area for size quantification, (b) Results of spatial kurtosis

\section{Conclusion and future work}

This work focusses on detection and evaluation of the CFRP-epoxy adhesive single-lap joints with artificially embedded contaminations of different materials in separate samples using eddy current pulse-compression thermography. Three processing steps have been applied: (1) the removal of non-uniform heating through PLS; (2) sliding Kernel Principal Component Analysis for determination of the best time interval to reveal information about contamination layer; (3) classification of contamination properties at the specific time using proposed features. The conclusions are as follows:

1. PLS can remove the non-uniform heating pattern and achieve better results of perfect bond subtraction because it solves the possible problem of misalignment of spatial coordinates resulting from different experiments.

2. After removal of non-uniform heating pattern, sliding K-PCA features and norm of principle component can be applied for detection of contamination layer in heating and cooling stages. Since eddy current can generate volumetric Joule heating in CFRP, the first time instant of the high contrast happens at the very beginning of the retrieved impulse responses, i.e. first 15 frames $(300 \mathrm{~ms})$, indicating the difference in contamination's electrical properties on adhesive joints. It has also been verified that 60th frame contains the maximum information about the contaminations in the cooling stage;

3. The contamination proprieties can be classified using the derivatives of impulse response at specific time. The release agent contamination has the minimum influence on the electrical and thermal properties of the adhesive layer compared with brass film and release film. The release agent contamination affects the thermal properties of the adhesive epoxy layer more than the electrical properties.

This approach can address the non-uniform heating problem with complex geometry and improve SNR for the detection and characterization of contaminations. Future work will investigate the quantitative determination of electrical and thermal properties of the multilayer structures using eddy current thermography techniques.

\section{Acknowledgments}

This project is funded by the European Union's Horizon 2020 research and innovation programme under the Marie Skłodowska-Curie grant agreement No 722134 - NDTonAIR.

\section{Reference}

[1] Barile C, Casavola C, Pappalettera G, Vimalathithan PK. Characterization of adhesive bonded CFRP laminates using full-field digital image stereo-correlation and finite element analysis. Composites Science and Technology. 2019;169:16-25.

[2] Lim AS, Melrose ZR, Thostenson ET, Chou T-W. Damage sensing of adhesively-bonded hybrid composite/steel joints using carbon nanotubes. Composites Science and Technology. 2011;71:1183-9. 
[3] Casavola C, Palano F, De Cillis F, Tati A, Terzi R, Luprano V. Analysis of CFRP joints by means of T-pull mechanical test and ultrasonic defects detection. Materials. 2018;11:620.

[4] Heide-Jørgensen S, de Freitas ST, Budzik MK. On the fracture behaviour of CFRP bonded joints under mode I loading: Effect of supporting carrier and interface contamination. Composites Science and Technology. 2018;160:97-110.

[5] Muliana A, Nair A, Khan KA, Wagner S. Characterization of thermo-mechanical and longterm behaviors of multi-layered composite materials. Composites Science and Technology. 2006;66:2907-24.

[6] Tornow C, Schlag M, Lima LCM, Stübing D, Hoffmann M, Noeske P-LM, et al. Quality assurance concepts for adhesive bonding of composite aircraft structures-characterisation of adherent surfaces by extended NDT. Journal of Adhesion Science and Technology. 2015;29:2281-94.

[7] Palumbo D, Tamborrino R, Galietti U, Aversa P, Tatì A, Luprano V. Ultrasonic analysis and lock-in thermography for debonding evaluation of composite adhesive joints. NDT \& E International. 2016;78:1-9.

[8] Markatos D, Tserpes K, Rau E, Markus S, Ehrhart B, Pantelakis S. The effects of manufacturing-induced and in-service related bonding quality reduction on the mode-I fracture toughness of composite bonded joints for aeronautical use. Composites Part B: Engineering. 2013;45:556-64.

[9] Yang R, He Y. Optically and non-optically excited thermography for composites: A review. Infrared Physics \& Technology. 2016;75:26-50.

[10] Milovanović B, Banjad Pečur I. Review of active IR thermography for detection and characterization of defects in reinforced concrete. Journal of Imaging. 2016;2:11.

[11] Shin PH, Webb SC, Peters KJ. Pulsed phase thermography imaging of fatigue-loaded composite adhesively bonded joints. NDT \& E International. 2016;79:7-16.

[12] Waugh R, Dulieu-Barton JM, Quinn S. Pulse phase thermography and its application to kissing defects in adhesively bonded joints. Applied Mechanics and Materials: Trans Tech Publ; 2011. p. 369-74.

[13] Grosso M, Lopez JE, Silva VM, Soares SD, Rebello JM, Pereira GR. Pulsed thermography inspection of adhesive composite joints: computational simulation model and experimental validation. Composites Part B: Engineering. 2016;106:1-9.

[14] Waugh RC, Dulieu-Barton JM, Quinn S. Thermographic Identification of Defects in Adhesively Bonded Joints. Advancement of Optical Methods in Experimental Mechanics, Volume 3: Springer; 2014. p. 347-51.

[15] Ren W, Liu J, Tian GY, Gao B, Cheng L, Yang H. Quantitative non-destructive evaluation method for impact damage using eddy current pulsed thermography. Composites Part B: Engineering. 2013;54:169-79.

[16] Schlichting J, Maierhofer C, Kreutzbruck M. Crack sizing by laser excited thermography. NDT \& E International. 2012;45:133-40.

[17] Cheng L, Gao B, Tian GY, Woo WL, Berthiau G. Impact damage detection and identification using eddy current pulsed thermography through integration of PCA and ICA. IEEE Sensors Journal. 2014;14:1655-63.

[18] Liang T, Ren W, Tian GY, Elradi M, Gao Y. Low energy impact damage detection in CFRP using eddy current pulsed thermography. Composite Structures. 2016;143:352-61.

[19] Cheng L, Tian GY. Comparison of nondestructive testing methods on detection of delaminations in composites. Journal of sensors. 2012;2012.

[20] Yang R, He Y. Eddy current pulsed phase thermography considering volumetric induction heating for delamination evaluation in carbon fiber reinforced polymers. Applied Physics Letters. 2015;106:234103.

[21] He Y, Yang R. Eddy current volume heating thermography and phase analysis for imaging characterization of interface delamination in CFRP. IEEE Transactions on Industrial Informatics. 2015;11:1287-97.

[22] Zimnoch M, Oliferuk W, Maj M. Estimation of defect depth in steel plate using lock-in IR thermography. acta mechanica et automatica. 2010;4:106-9. 
[23] Tian GY, Gao Y, Li K, Wang Y, Gao B, He Y. Eddy current pulsed thermography with different excitation configurations for metallic material and defect characterization. Sensors. 2016;16:843.

[24] Gao Y, Tian GY, Wang P, Wang H, Gao B, Woo WL, et al. Electromagnetic pulsed thermography for natural cracks inspection. Scientific reports. 2017;7:42073.

[25] Li Z, Meng Z. A review of the radio frequency non-destructive testing for carbon-fibre composites. Measurement Science Review. 2016;16:68-76.

[26] Yi Q, Tian GY, Malekmohammadi H, Zhu J, Laureti S, Ricci M. New features for delamination depth evaluation in carbon fiber reinforced plastic materials using eddy current pulse-compression thermography. NDT \& E International. 2019;102:264-73.

[27] Xu C, Zhou N, Xie J, Gong X, Chen G, Song G. Investigation on eddy current pulsed thermography to detect hidden cracks on corroded metal surface. Ndt \& E International. 2016;84:27-35.

[28] Silipigni G, Burrascano P, Hutchins DA, Laureti S, Petrucci R, Senni L, et al. Optimization of the pulse-compression technique applied to the infrared thermography nondestructive evaluation. NDT \& E International. 2017;87:100-10.

[29] Lopez F, Ibarra-Castanedo C, de Paulo Nicolau V, Maldague X. Optimization of pulsed thermography inspection by partial least-squares regression. Ndt \& E International. 2014;66:128-38.

[30] Yin A, Gao B, Yun Tian G, Woo W, Li K. Physical interpretation and separation of eddy current pulsed thermography. Journal of Applied Physics. 2013;113:064101.

[31] Zeng Z, Zhou J, Tao N, Feng L, Zhang C. Absolute peak slope time based thickness measurement using pulsed thermography. Infrared Physics \& Technology. 2012;55:200-4. 